\title{
Open-Nasality and Eustachian tube Dysfunction After Acute Weight Loss in Bariatric Surgery
}

Case

Report

\author{
Hatem Ezzeldin Hassan ${ }^{1}$ and Akram Abdelrahman ${ }^{2}$
}

Department of Otorhinolaryngology, Faculty of Medicine, ${ }^{1}$ Sohag University,

${ }^{2}$ Mansoura University, Egypt

\begin{abstract}
Velopharyngeal Insufficiency (VPI) means that the velopharyngeal closure is inadequate or disturbed. VPI may be organic or functional and results in open nasality. VPI due to sudden weight loss is a very rare condition. We reported a case of open nasality and Eustachian tube dysfunction due to sudden weight loss after bariatric surgery.
\end{abstract}

Key Words: Bariatric surgery, eustachian tube dysfunction, open nasality, velopharyngeal insufficiency, weight loss.

Received: $23^{\text {th }}$ April 2019, Accepted: 07 ${ }^{\text {th }}$ September 2019

Corresponding Author: Hatem Ezzeldin Hassan, M.D., Department of Otorhinolaryngology, Faculty of Medicine, Sohag University, Sohag, Egypt, Tel.: +966 594183458, E-mail: hezzm268@yahoo.com

ISSN: 2090-0740, March 2020 Vol. 21, No. 1.

\section{INTRODUCTION}

Velopharyngeal dysfunction (VPD) describes a set of disorders resulting in the leakage of air into the nasal passages during speech production. (VPD) refers to any situation in which an individual is unable to completely close the nasal airway during speech. Anatomic causes are most common and are typically associated with a previously repaired cleft palate. Often referred to as velopharyngeal insufficiency (VPI), the soft palate may be too short or "insufficient" to permit adequate approximation of the velum to the posterior pharynx. Neuromuscular etiologies can also result in VPD and are occasionally referred to as velopharyngeal incompetence ${ }^{[1]}$.

Bariatric surgery (weight loss surgery) is one of the fastest growing surgical discipline. Bariatric operations, as any surgical procedure, may result in complications and one of the inevitable complications is permanent alteration of a patient's anatomy, which can lead to complications at any time during the course of a patient's life ${ }^{[2]}$.

Reviewing literature, and to our knowledge, no articles reported open nasality after bariatric surgery.

\section{CASE REPORT}

A 42 years old female patient presented to phoniatrics clinic complaining of unexplained nasal tone of speech and ear fullness of rapid onset and progressive course for nine months. The nasal tone of speech was noticed by surrounding listeners. The patient was also complaining of as intermittent ear discomfort and sensation of water in both ears. The patient underwent bariatric surgery due to morbid obesity one year ago. After surgery, sudden weight loss occurred quickly and no little warning. The weight of the patient before the operation was one hundred and two $\mathrm{kg}$ and body mass index (BMI) was (40.8). She started to lose weight after surgery rapidly and instead of as after nine month her weight was $48 \mathrm{~kg}$ and BMI was (19.2). The former symptoms started to appear 3 months after the operation. The patient had no other medical or surgical history of clinical importance.

\section{On examination}

The palatal length was adequate with good palatal mobility. The velopharyngeal valve (VPV) was sufficient with fair lateral pharyngeal walls movements.

Auditory perceptual assessment of speech: Mild to moderate open nasality with no compensatory mechanisms or articulation errors.

\section{Clinical Diagnostic Tests}

(A/I and cold mirror tests) were positive for both vowels and consonants.

\section{Additional Diagnostic Aids}

1. Nasometer: Nasalance score for oral sentence was 25.8 $\%$, for nasal sentence was 61.4 and that for oronasal text was 49.2 . (normal values are $10.9 \%, 55.8 \%$ and $33.8 \%$, the oral text and the nasal text respectively according to Van lierde etal, $\left.{ }^{[9]}\right)$.

2. Fiberoptic nasolaryngoscope showed mobile soft palate with relative contribution of posterior and lateral pharyngeal walls with coronal closure of VPV (Figure 1). Slight insufficiency and bubbling of air on either sides of VPV were bserved for fricative sounds (Figure 2). 


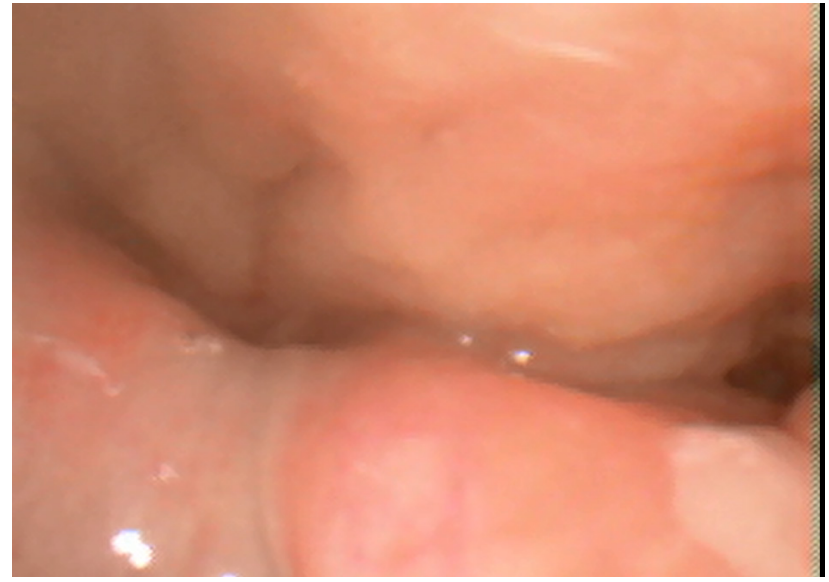

Fig. 1: Coronal closure with insufficient VPV

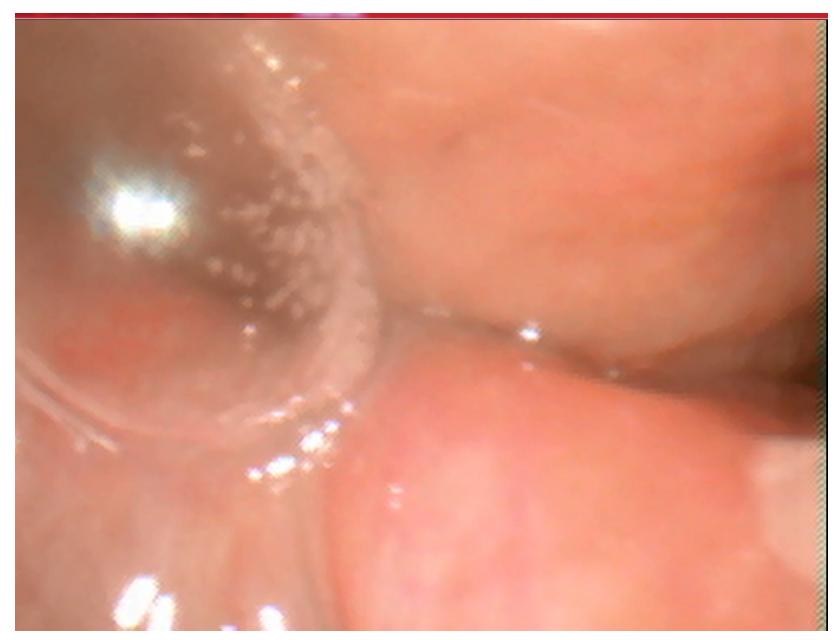

Fig. 2: VPI with air bubbling during vocalization

Basic audiological evaluation: Bilateral normal hearing sensitivity with normal middle ear functions.

\section{DISCUSSION}

Bariatric surgeries are associated with anatomical changes in the body due to weight loss. Studies ${ }^{[3,4,5]}$ suggested changes in upper airway dimensions and decrease parapharyngeal pad of fat in obese subjects after acute weight loss.

Shahid et $a l^{[3]}$ studied the effect of weight loss on the pharynx dimensions. They examined the patients (after weight loss) by a low-dose CT scan of the upper airway using a 16-slice scanner. They found increase in velopharyngeal airway volume and significant decrease in parapharyngeal pad of fat which resulted in widening of Velopharyngeal valve surface area.

Liu, et $a{ }^{[10]}$ measured pharynx dimensions in normal subjects by MRI and ultrasound. They found that neck circumference, $\mathrm{cm}(37.6 \pm 3.7)$ and Lateral pharyngeal wall thickness, $\mathrm{cm}(3.8 \pm 0.6)$

Regarding the described case our suggestion is that decrease thickness of lateral pharyngeal walls and parapharyngeal fat absorption occurred because of sudden weight loss after bariatric surgery. Sudden loss of lateral pharyngeal walls thickness and parapharyngeal pad of fat left gaps on either side of VPV resulted in open nasality and leakage of air through VPV during speech production particularly with fricatives (/s/ and /S/ sounds).

Nasoendoscopic evaluation is a procedure in which a flexible endoscope is inserted through the inferior meatus and located the upper part of velopharyngeal port to provide visualizing the velopharyngeal mechanism while the patient phonates a sample speech. Nasoendoscopy provides the information of the size and location of velopharyngeal gap and relative contribution of the velum, lateral pharyngeal wall and posterior pharyngeal wall while velopharyngeal port is closing ${ }^{[6]}$.

The three basic closure patterns of velopharyngeal port closure are as follows: (1) coronal, the posterior movement of the velum is mainly contributed to close of velopharyngeal port, with minorly medial displacement of lateral pharyngeal wall; (2) sagittal, the medial displacement of lateral pharyngeal wall is mainly contributed to close of velopharyngeal port, with minorly posterior movement of the velum; (3) circular, the posterior movement of the velum and the pharyngeal wall contribute to close of velopharyngeal port ${ }^{[6]}$.

In the current case the velopharyngeal port closure was coronal with dominant movement was at the soft palate and little movement of the lateral pharyngeal walls. There was VPI with escape of air during vocalization observed as bubbling of air especially for fricative sounds.

In the current case, Eustachian tube dysfunction was reported by the patient as intermittent ear fullness, ear discomfort and sensation of water in both ears. The present findings are suggestive of Eustachian tube dysfunction due to loss of fat around Eustachian tube related to sudden weight loss after bariatric surgery. Basic audiological evaluation reflected normal results, however Eustachian tube dysfunction is mostly diagnosed clinically depending on the symptoms of the patients.

Dysfunction of the Eustachian tube was previously reported by Pascoto et $a l^{[7]}$ as a result of sudden weight loss after bariatric surgery. They found $26.3 \%$ of patients presented by aural fullness, sensation of water in the ears and ticking or popping sounds.

Patulous Eustachian tube was found in $21.28 \%$ of the patients after rapid weight loss because of rapid loss of adipocyte tissue around the tube ${ }^{[8]}$. In the present case no signs of patulous Eustachian tube were observed.

\section{CONCLUSION}

Acute weight loss after Bariatric surgery may result in velopharyngeal Insufficiency and open nasality as well as Eustachian tube dysfunction.

\section{CONFLICT OF INTEREST}

No conflict of interest to declare. 


\section{REFERENCES}

1. Shin YJ, Kim Y. Investigation of postoperative hypernasality after superiorly based posterior pharyngeal flap. Maxillofac Plast Reconstr Surg. 2018;40(1):23. Published 2018 Aug 30.

2. Lim R, Beekley A, Johnson DC, Davis KA. Early and late complications of bariatric operation. Trauma Surg Acute Care Open. 2018;3(1).

3. Shahid ML, Chitiboi T, Ivanovska T, Molchanov V, Völzke H, Linsen L. Automatic MRI segmentation of para-pharyngeal fat pads using interactive visual feature space analysis for classification. BMC Med Imaging. 2017;17(1):15.

4. Aylin Yucel, Mehmet Unlu, Alpay Haktanir, Murat Acar and Fatma Fidan Evaluation of the Upper Airway Cross-sectional Area Changes in Different Degrees of Severity of Obstructive Sleep Apnea Syndrome: Cephalometric and Dynamic CT Study, American Journal of Neuroradiology November 2005, 26 (10) 2624-2629;

5. Pahkala R1, Seppä J, Ikonen A, Smirnov G, Tuomilehto H.The impact of pharyngeal fat tissue on the pathogenesis of obstructive sleep apnea. Sleep Breath. 2014 May;18(2):275-82.

6. Seung Min NamSurgical treatment of velopharyngeal insufficiency. Arch Craniofac Surg. 2018;19(3): 163-167.

7. Pascoto G, Abreu C, Silva ML, Weber R, Pignatari SS, Stamm A. The impact of acute loss of weight on eustachian tube function. Int Arch Otorhinolaryngol. 2014;18(4):376-9.

8. Muñoz D, Aedo C, Der C. Patulous eustachian tube in bariatric surgery patients. Otolaryngol Head Neck Surg. 2010;143(4):521-524

9. Van Lierde KM, Wuyts FL, De Bodt M, Van Cauwenberge P. Nasometric values for normal nasal resonance in the speech of young flemish adults. Cleft Palate-Craniofacial Journal. 2001;38(2):112-118.

10. Liu, K. H., Chu, W. C., To, K. W., Ko, F. W., Tong, M. W., Chan, J. W., \& Hui, D. S. (2007). Sonographic measurement of lateral parapharyngeal wall thickness in patients with obstructive sleep apnea. Sleep, 30(11), 1503-8. 